DOI: 10.12731/2070-7568-2021-10-1-70-81

УДК 332.02

\title{
ИНСТРУМЕНТЫ МЕНЕДЖМЕНТА КАК ФАКТОРЫ ЭФФЕКТИВНОГО РАЗВИТИЯ МАЛОГО И СРЕДНЕГО ПРЕДПРИНИМАТЕЛЬСТВА
}

\section{Ловкова Е.С., Пенья М.}

Для расширения малого и среднего бизнеса и усиления внутреннего контроля над ним, важно полагаться на инструменты управления, которые помогут принимать более обоснованные решения $и$ стимулировать развитие деятельности организации малого предпринимательства.

Цель работы. В статье составить список основных инструментов менеджмента, используемых руководителями малого и среднего предпринимательства. Выявить основные ошибки препятствующие использованию современных инструментов менеджмента и предложить мероприятия по их устранению.

Метод или методология проведения работы: был использован подход позволяющий комплексно исследовать характеристики инструментов менеджмента и эффективного развития малого и среднего предпринимательства.

Результаты: выделены основные ошибки, препятствующие использованию инструментов менеджмента и таким образом тормозящие развитию предприятий малого и среднего бизнеса. Таким образом, актуальность научного исследования на тематику инструменты менеджмента влияет на эффективность развития малого и среднего предпринимательства.

Область применения результатов: полученные результаты целесообразно использовать предпринимателям малого и среднего бизнеса.

Ключевые слова: эффективное развитие организации; инструменты менеджмента; предпринимательство. 


\section{MANAGEMENT TOOLS AS FACTORS OF EFFECTIVE DEVELOPMENT OF SMALL AND MEDIUM ENTREPRENEURSHIP}

\section{Lovkova E.S., Penha M.}

To expand small and medium-sized businesses and strengthen internal control over them, it is important to rely on management tools that will help make more informed decisions and stimulate the development of small business organizations.

The purpose of the work. In the article to make a list of the main management tools used by managers of small and medium-sized businesses. Identify the main errors that prevent the use of modern management tools and suggest measures to eliminate them.

Method or methodology of the work: an approach was used to comprehensively investigate the characteristics of management tools and the effective development of small and medium-sized businesses.

Results: the main errors that hinder the use of management tools and thus hinder the development of small and medium-sized businesses are identified. Thus, the relevance of scientific research on the subject of management tools affects.

Keywords: effective development of the organization; management tools; entrepreneurship.

\section{Введение}

Малое предпринимательство в странах «третьего мира» составляет до 95\% общего экономического потенциала, в европейских странах 45-70\%, в США более 50\% населения включен в сектор малого бизнеса. В России малое предпринимательство сформировалось как отдельный сектор экономик сравнительно недавно. Особенностью развития российского малого предпринимательства главным образом являются географический и национальный признаки.

Доля малого и среднего бизнеса в экономике России составляет $21,9 \%$ или более 20 трлн руб. в денежном эквиваленте. 
По данным проекта «СберДанные» по состоянию на конец I квартала 2019 года, 18,3 млн рабочих мест в России, или четверть $(25,6 \%)$, создали малый и средний бизнес, $18,6 \%$ приходится на юридических лиц и 7\% - на индивидуальных предпринимателей. «Более половины занятых в МСП сконцентрированы всего в 10 регионах России. Более 20\% приходится на Москву, еще $8 \%$ - на Санкт-Петербург», - говорится в исследовании.

Роль малого предпринимательства в развитии экономики страны нельзя недооценить, именно за счет поддержки данного сектора правительство может решить общие проблемы роста благосостояния населения страны, а также повышение процентного соотношения людей со средним уровнем доходов. Таким образом, можно говорить о том, что развитие малого предпринимательства напрямую связано с повышением уровня качества жизни населения страны.

Цель работы: составить список основных инструментов менеджмента, используемых руководителями малого и среднего предпринимательства. Выявить основные ошибки препятствующие использованию современных инструментов менеджмента и предложить мероприятия по их устранению.

\section{Метод или методология проведения работы}

Информационной базой исследования являются современные инструменты менеджмента используемые в экономических системах. В работе использован подход позволяющий комплексно исследовать характеристики инструментов менеджмента и эффективного развития малого и среднего предпринимательства. Он позволяет значительно расширить представление о сущности и тенденциях развития организации, всесторонне раскрыть содержание происходящих в ней процессов, выявить объективные закономерности формирования этой многоаспектной системы.

\section{Результаты исследования и их обсуждение}

Для эффективной работы в современных условиях руководителя малого и среднего предпринимательства необходимо пользоваться 
различными инструментами управления. Список основных инструментов менеджмента, используемых субъектами малого и среднего предпринимательства:

1. Система управления человеческими ресурсами, объединяет различные процессы, которые влияют на работников и организацию в целом.

Это позволяет оптимизировать и централизовать ключевые процессы в организации, связанные с сотрудниками, такие как:

- Подбор персонала

- Обучающие программы

- Анализ производительности и компенсация

- Управление контрактами и условиями труда

Эти системы повышают продуктивность деятельности организации, упрощая мониторинг производительности труда сотрудников и задач, которые они выполняют.

Таким образом, работодатель может иметь доступ в режиме реального времени к информации, связанной с работой сотрудников, и, следовательно, принимать эффективные решения и своевременно координировать работу сотрудников.

1.2. Менеджер социальных сетей. С помощью интернет платформ организация может коммуницировать с широким сообществом пользователей и даже продавать свою продукцию напрямую.

Социальные сети являются мощным отделом продаж, который увеличивает коэффициент конверсии учреждений на 50\%, то есть процент пользователей, совершающих покупки, бронирование, регистрацию или загрузку. По данным АКИТ ${ }^{1}$ доля онлайн-продаж, которые приходятся на социальные сети, составляет почти 20\% от общего объёма рынка интернет-торговли в России за 2018 г.

Этот инструмент помогает планировать, организовывать и опубликовывать необходимый рекламный материал, что будет способствовать активному развитию организации в мире 2.0.

1.3. Программное обеспечение CRM. Система управления взаимоотношениями с клиентами или CRM-система - это инструменты управления, которые помогут создать базу клиентов и управлять отношениями

$1 \mathrm{http}: / / \mathrm{vc} . \mathrm{ru}$ 
с ними. Эта система собирает информацию о пользователях, которая позволит организации создавать ссылки и напрямую связываться с ней, например, номера телефонов, социальные сети и электронную почту.

Такие данные, хранящиеся в централизованной базе данных, особенно полезны для отдела продаж, отдела обслуживания клиентов и поддержки, а также для тех, кто отвечает за маркетинг.

1.4. ERP система, представляет собой организационную стратегию интеграции производства и операций, управления трудовыми ресурсами и финансами организации. Данный программный продукт ориентирован на сбалансированность и оптимизацию всех ресурсов (трудовых, материальных, нематериальных, финансовых и тд.) организации. Несомненно, ERP вносит большой вклад в развитие и оптимизацию малых и средних организаций. Это связано с тем, что система имеет широкую сферу применения за счет централизации всей необходимой информации организации и, следовательно, повышает эффективность процессов и операций в организации.

Система ERP обеспечивает три преимущества:

- Упрощает бухгалтерский учет. Благодаря этому инструменту можно мгновенно получить финансовые отчеты, а, следовательно, выполнение финансовых обязательств в организации станет намного проще.

Кроме того, при доступе к системе руководитель может видеть обновленные актуальные отчеты, которые помогут принимать более эффективные решения. Руководитель может наблюдать за общим доходом, затратами и расходами, а также за деталями каждой из этих переменных.

- Контролирует уровень запасов. Отсутствие запасов на складе, также как и полная загрузка склада может привести к операционным проблемам. Благодаря специализированному модулю можно просматривать в режиме реального времени уровень запасов продукции во всех филиалах и складах, в которых они находятся. ERP позволяет отслеживать путь товаров от входа на склад до доставки клиенту.

- Оптимизировать покупки. ERP дает возможность увидеть, на что потрачены денежные средства и когда, поскольку система мгновенно фиксирует расходы, загружая счет-фактуру постав- 
щика и списывая его на счет организации. Эта функция также поможет оптимизировать тайм-менеджмент в организации.

Не смотря на большой круг инструментов управления и программного обеспечения, на сегодняшней день почти каждый пятый предприниматель малого и среднего бизнеса терпит неудачу. Задача укрепления экономики страны, помимо стимулирования создания новых предприятий, заключается в их укреплении. Поэтому важно, чтобы предприниматели знали типичные ошибки, допущенные при запуске проекта, которые ставят под угрозу стабильность и выживание своего бизнеса. Рассмотрим распространенные ошибки, мешающие развитию малого бизнеса.

- Отсутствие планирования. Наличие бизнес-плана помогает визуализировать целевой рынок, добавленную стоимость предлагаемого продукта или услуги, какие каналы распространения и маркетинга являются идеальными, а также устанавливать бюджеты и процессы для облегчения работы и т. д.

- Отсутствие видения. Это означает придерживаться первоначальной стратегии работы и не адаптировать ее к финансовым результатам, которые компания получает в первый год деятельности, и к тому, что диктует рынок.

- Отсутствие образования и нежелание обучаться. Без необходимых знаний в различных отраслях, таких как юридическая, финансовая, налоговая и операционная, рост бизнеса не может быть реализован.

- Плохое управление. Это причина, по которой 43\% организаций малого предпринимательства закрываются, по данным Национальной комиссии по защите пользователей финансовых услуг (Condusef). Вот почему важно умение управлять капиталом, устанавливать заработную плату, контролировать денежный поток и т. д.

- Отсутствие финансового контроля. Ведение учета расходов и доходов ежедневно необходимо для принятия правильных управленческих решений.

- Отсутствие стратегического анализа. Отсутствие точной, надежной информации в режиме реального времени, указывающей на эффективность компании, приведет к неправильному принятию решений. 
- Отсутствие знаний о системе налогообложения. Необходимо знать и понимать налоговые положения, которые должны соблюдаться после начала операций, такие как выставление счетов, выдача цифровых квитанций о заработной плате и отправка электронного учета в орган и т.д.

С самого начала руководитель организации должен очень четко понимать цели, миссию и видение своего бизнеса, и после вывода бизнеса на рынок крайне важно контролировать и оценивать все административные, бухгалтерские, финансовые и операционные процессы, а также измерять эффективность каждого шага, который предпринимается, чтобы действовать правильно.

Для устранения этих ошибок предлагается использовать услуги многофункциональных региональных центров, инжиниринговых центров и бизнес-инкубаторов которые предлагают:

- специальные образовательные программы, помогающие развивать управленческие навыки у начинающих предпринимателей;

- обучающие мероприятия, направленные на повышение квалификации персонала;

- консультации по правовым вопросам, информационному сопровождению деятельности, трудовому законодательству, финансовому планированию и налогам, маркетинговому сопровождению деятельности, началу ведения собственного дела;

- обучающие тренинги и семинары;

- участие в выставках и конкурсах.

В результате чего повыситься уровень квалификации и образованности руководителя организации и персонала, их осведомленность о новом программном обеспечении, что позволит им использовать в своей работе более новые инструменты управления.

\section{Заключение}

Технология перестала быть роскошью или привилегией во всем мире, ее использование стало фундаментальным элементом в личной и деловой сфере. В таком активном и глобализованном мире компании должны быть быстрыми и эффективными со всеми своими ресурсами, применение технологий необходимо для решения проблем и устранения барьеров организации, с помощью иннова- 
ционных систем, которые адаптируются к потребностям каждой из них. То, что раньше занимало недели и даже месяцы, сегодня можно закончить за несколько минут и без каких-либо дополнительных усилий или осложнений.

Когда компания сопротивляется внедрению новых технологий в каждый из своих процессов, она увеличивает свои шансы на стагнацию и отставание, особенно те, которые находятся на стадии роста, которые еще не полностью стабилизированы или не заняли определенную долю на рынке.

Вот почему растущие компании должны каждый день бороться за то, чтобы идти рука об руку с технологическими достижениями и адаптироваться к ним, чтобы ускорить свои процессы и, конечно же, поддерживать конкурентоспособность на рынке.

\section{Список литературы}

1. Герасимов Б.Н., Герасимов К.Б. Инструменты обеспечения технологий решения задач управления организации // Управленческие науки. 2018. №1. С. 82-89. https://doi.org/10.26794/2304022Х-2018-8-1-82-89

2. Ильина О.Н. Методология управления проектами: становление, современное состояние и развитие: монография. Москва: Вузовский учебник: ИНФРА-М, 2019. 208 с.

3. Калинченко М.П. Устойчивое развитие предприятия: оценка, проект, функциональная стратегия маркетинг менеджмента // Вестник Астраханского государственного технического университета. Серия: Экономика. 2019. №1. С. 40-52. https://doi.org/10.24143/2073-55372019-1-40-52

4. Ловкова Е.С., Корнев А.В. Государственная поддержка малого предпринимательства и выявление проблем развития // Бюллетень науки и образования. 2019. Т. 5, №5. С. 324-330. https://doi.org/10.33619/2414$2948 / 42 / 42$

5. Ловкова Е.С., Тимофеева Е.С., Савельев И.И. Проблемы и особенности развития малого предпринимательства в России // Экономика и управление: проблемы, решения, 2018. Т. 4, №4. С. 83-88. 
6. Проект «Малый бизнес и поддержка индивидуальной предпринимательской инициативы» [Электронный ресурс] // Федеральный портал малого и среднего предпринимательства. М., 2017. http:// smb.gov.ru/mediacenter/bisnessnews/17791.htm

7. Резник С.Д. Дороги к менеджменту: о себе и окружавших меня людях, о времени и событиях. Москва: ИНФРА-М, 2021. 526 с.

8. Рыжкова Е. Семь ошибок предпринимателей, которые приведут бизнес к провалу // RUSBASE. 2019. https://rb.ru/opinion/oshibki-biznesa/

9. Салимова Т.А., Гудкова Д.Д. Инструментарий оценки устойчивого развития организации // Научно-технические ведомости СПбГПУ. Экономические науки. 2017. Т. 10, №5. C. 151-160. https://economy. spbstu.ru/article/2017.67.14/

10. Современные проблемы менеджмента: монография / под общ. ред. д-ра экон. наук, проф. С.Д. Резника. М.: ИНФРА-М, 2018. 243 с.

11. Трансформация бизнеса в условиях рыночной нестабильности : монография / под ред. Н. К. Моисеевой. Москва: КУРС: ИНФРА-М, 2019. $416 \mathrm{c}$.

12. Шумков М.А. Новые тенденции в развитии системы управления // Молодой ученый. 2020. № 15 (305). C. 373-375. https://moluch.ru/ archive/305/68834/

13.Lovkova E.S., Mihailov Y.Y Application of the cluster approach in the development of small entrepreneurship in the region // ACM International Conference Proceeding Series. Proceedings Papers - 3rd International Scientific and Practical Conference, DEFIN 2020. https:// doi.org/10.1145/3388984.3390815

14. Darrell Rigby, Barbara Bilodeau. Management tools and trends. Bain \&Company, 2018. https:/www.bain.com/contentassets/f8361c5cd99e4 f40bbbf83c17d6a91b9/bain_brief-management_tools_and_trends.pdf

\section{References}

1. Gerasimov B.N., Gerasimov K.B. Instrumenty obespecheniya tekhnologiy resheniya zadach upravleniya organizatsii [Tools for Providing Technologies to Solve Organizational Management Problems]. Upravlencheskie nauki [Management science], 2018, no. 1, pp. 82-89. https:// doi.org/10.26794/2304-022X-2018-8-1-82-89 
2. Ilina O.N. Metodologiya upravleniya proektami: stanovlenie, sovremennoe sostoyanie i razvitie [Project management methodology: formation, current state and development]: monograph. Moscow: Vuzovskiy uchebnik: INFRA-M, 2019. 208 p.

3. Kalinchenko M.P. Ustoychivoe razvitie predpriyatiya: otsenka, proekt, funktsional'naya strategiya marketing menedzhmenta [Sustainable development of the enterprise: assessment, project, functional strategy of marketing management]. Vestnik of Astrakhan State Technical University. Series: Economics, 2019, no. 1, pp. 40-52. https://doi.org/10.24143/20735537-2019-1-40-52

4. Lovkova E.S., Kornev A.V Gosudarstvennaya podderzhka malogo predprinimatel'stva i vyyavlenie problem razvitiya [State support of micro-entrepreneurship and identify the development problems]. Byulleten nauki i obrazovaniya [Bulletin of Science and Education], 2019, vol. 5, no. 5, pp. 324-330. https://doi.org/10.33619/2414-2948/42/42

5. Lovkova E.S., Timofeeva E.S., Saveliev I.I. Problemy i osobennosti razvitiya malogo predprinimatel'stva v Rossii [Problems and Features of Small Business Development in Russia]. Ekonomika i upravlenie: problemy, resheniya [Economics and Management: Problems, Solutions], 2018, vol. 4, no. 4, pp. 83-88.

6. Proekt «Malyy biznes i podderzhka individual'noy predprinimatel'skoy initsiativy» [Project "Small business and support for individual entrepreneurial initiative"]. Federal portal of small and medium-sized businesses. M., 2017.http://smb.gov.ru/mediacenter/bisnessnews/17791.htm

7. Reznik S.D. Dorogi kmenedzhmentu: o sebe i okruzhavshikh menya lyudyakh, $o$ vremeni $i$ sobytiyak [Roads to management: about myself and the people around me, about time and events]. Moscow: INFRA-M, 2021. 526 p.

8. Ryzhkova E. Sem' oshibok predprinimateley, kotorye privedut biznes k provalu [Seven mistakes of entrepreneurs that will lead business to failure]. RUSBASE. 2019. https://rb.ru/opinion/oshibki-biznesa/

9. Salimova T.A., Gudkova D.D. Instrumentariy otsenki ustoychivogo razvitiya organizatsii [Toolkit for assessing the sustainable development of an organization]. Nauchno-tekhnicheskie vedomosti SPbGPU. Ekonomicheskie nauki [Scientific and technical statements of SPbSPU. Economic 
sciences], 2017, vol. 10, no. 5, pp. 151-160. https://economy.spbstu.ru/ article/2017.67.14/

10. Sovremennye problemy menedzhmenta [Modern problems of management]: monograph / ed. S.D. Reznik. Moscow: INFRA-M, 2018.243 p.

11. Transformatsiya biznesa v usloviyakh rynochnoy nestabil'nosti [Business transformation in conditions of market instability]: monograph / ed. N.K. Moiseeva. Moscow: KURS: INFRA-M, 2019. 416 p.

12. Shumkov M.A. Novye tendentsii v razvitii sistemy upravleniya [New tendencies in the development of the control system]. Molodoy ucheny [Young scientist], 2020, no. 15 (305), pp. 373-375. https://moluch.ru/ archive/305/68834/

13. Lovkova E.S., Mihailov Y.Y Application of the cluster approach in the development of small entrepreneurship in the region. ACM International Conference Proceeding Series. Proceedings Papers - 3rd International Scientific and Practical Conference, DEFIN 2020. https://doi. org/10.1145/3388984.3390815

14. Darrell Rigby, Barbara Bilodeau. Management tools and trends. Bain \& Company, 2018. https://www.bain.com/contentassets/f8361c5cd99e4f40bbbf83c17d6a91b9/bain_brief-management_tools_and_trends.pdf

\section{ДАННЫЕ ОБ АВТОРАХ}

Ловкова Елена Сергеевна, к.э.н., доцент, доцент кафедры «Менеджмент и маркетинг» ФУБОУ ВО «Владимирский государственный университет им. А.Г. и Н.Г. Столетовых» ул. Горького, 87, г. Владимир, 600000, Российская Федераџия nikishinaes@yandex.ru

Пенья Зелада Мартин Леонардо, магистр кафедры «Менеджмент и маркетинг» ФУБОУ ВО «Владимирский государственный университет им. А.Г. и Н.Г. Столетовых» ул. Горького, 87, г. Владимир, 600000, Российская Федераџия martinpenhazeladaleonardo@gmail.com 


\section{DATA ABOUT THE AUTHORS}

Lovkova Elena Sergeevna, Candidate of Economic Sciences, Associate Professor of the Department of Management and Marketing Vladimir State University named after A.G. and N.G. Stoletovs 87, Gorky Str., Vladimir, 600000, Russian Federation nikishinaes@yandex.ru ORCID: 0000-0002-9801-8860

Penha Zelada Martin Leonardo, Master of Management and Marketing Department Vladimir State University named after A.G. and N.G. Stoletovs 87, Gorky Str., Vladimir, 600000, Russian Federation martinpenhazeladaleonardo@gmail.com 